\section{Genetic Relationships among Native Species and Hybrid Cultivars of Asian Dendrobium (Orchidaceae) Using Amplified Fragment Length Polymorphism Markers}

\author{
Gen-Fa Zhu' ${ }^{1}$ and Dong-Mei Li \\ Guangdong Key Laboratory of Ornamental Plant Germplasm Innovation \\ and Utilization, Floricultural Research Institute, Guangdong Academy of \\ Agricultural Sciences, Guangzhou 510640, China
}

Additional index words. pedigree, Dendrobium, molecular identification, molecular phylogeny, morphological classification

\begin{abstract}
This study addresses the phylogenetic relationships among native species and hybrid cultivars of Asian Dendrobium by amplified fragment length polymorphism (AFLP). The plant materials of this study are composed of 37 accessions belonging to native species in China and 63 accessions proposed to be hybrid cultivars originating from Japan and Korea. Eight AFLP primer combinations produced a total of 1658 fragments with an average of 207 fragments per primer pair, of which 1655 bands were polymorphic. Specific AFLP markers were identified in 29 of 100 tested Dendrobium accessions. Unweighted pair group method based on arithmetic average (UPGMA) analysis was performed on Dice's similarity coefficient matrix and also average similarity of each species and cultivar. The tested 100 Asian Dendrobium accessions were grouped into seven clusters with the similarity coefficient of 0.49 . A first cluster consisted of 63 hybrid cultivars, 17 species of section Dendrobium, one species of section Formosae, and one species of section Callista. A second, fourth, and seventh cluster included five, three, and two species of section Dendrobium, respectively. A third group comprised five species of section Formosae. A fifth and sixth cluster contained three and two species of section Callista, respectively. These results indicated that the genetic relationships among tested Asian Dendrobium accessions were related to their origins, morphological classification, flower color, and pedigree, to some extent.
\end{abstract}

The genus Dendrobium is one of the largest genera in Orchidaceae family, comprising $\approx 1200$ species (http://en.wikipedid.org/ wiki/list_of_Dendrobium_species) of Dendrobium distributed in tropical and subtropical Asia and Oceania. They have been used not only in traditional Chinese medicine in Asian countries (Bulpitt et al., 2007), but also in commercial cut flowers and potted plants (Chen and Tsi, 2000). For commercial production of cut flowers and potted plants with new floral characteristics and other beneficial traits, a large number of novel cultivars of Dendrobium have been produced through interspecific hybridization in Japan and Korea. In China, there are 74 native species and two varieties of Dendrobium classified into 12 sections by traditional characteristics, including Grastidium (four species), Callista

\footnotetext{
Received for publication 18 Aug. 2010. Accepted for publication 1 Nov. 2010.

This research was financially supported by the Scientific and Technological Key Projects of Guangdong Province (No. 2003A2010401 and 2006A20201001) and the Scientific and technological Project of China (No. 2006BAD01A18).

${ }^{1}$ To whom reprint requests should be addressed; e-mail genfazhu@163.com.
}

(six species), Dendrobium (36 species), Distichophyllum (one species), Breviflores (two species), Stuposa (one species), Pedilonum (three species), Formosae (seven species), Stachyobium (five species), Crumenatae (four species), Aporum (three species), and Strongyle (two species) (Tsi et al., 1999). Therefore, these species of Dendrobium should be exploited for breeding.

It is important for breeding to understand the genetic relationships and molecular phylogeny of the genus Dendrobium. In recent years, genetic relationships and molecular identification of Dendrobium species have been measured by different molecular tools, including chloroplast DNA (Yukawa et al., 1993, 1996), internal transcribed spacer (ITS) sequences of nuclear ribosomal DNA and plastid mat $\mathrm{K}$ (Burke et al., 2008; Lau et al., 2001; Tsai et al., 2004; Wongsawad et al., 2001; Yuan et al., 2009; Yukawa, 2001), simple sequence repeat (SSR) marker (Gu et al., 2007; Yue et al., 2006), and intersimple sequence repeat (ISSR) marker (Wang et al., 2009). However, much information related to taxonomy and phylogenesis of the Dendrobium genus still needs to be solved. Hence, there is a need to study the genus Dendrobium using more abundant and reliable characters such as AFLP markers. The AFLP analysis provides a powerful DNA fingerprinting technique for DNAs of any origin or complexity, needs no cloning and sequencing, and produces a large number of recordable fragments, which enhance its power to detect polymorphism (Vos et al., 1995). The AFLP technique had been successfully used in the estimation of genetic relationships and differentiation among individuals, populations, and species of a wide range of plant families (e.g., Depypere et al., 2009; Karimi et al., 2009; Pamidiamarri et al., 2009; Seehalak et al., 2006). In particular, the genetic diversity and population structure of $D$. officinale was identified by AFLP (Li et al., 2008).

In the present study, we collected 37 native species of Dendrobium in China and 63 hybrid cultivars of Dendrobium originated from Japan and Korea. The pedigree of some hybrid cultivars, which was obtained by searching the web (http://apps.rhs. org.uk/horticulturaldatabase/orchidregister/ orchidregister.asp), was displayed in Supplemental Figure 1. The main objectives of this research were: 1) to test the AFLP technique for molecular identification of collected Asian Dendrobium; 2) to assess phylogenetic relationships among tested native species and hybrid cultivars of Asian Dendrobium; and 3) to supply molecular evidence for classification of Asian Dendrobium.

\section{Materials and Methods}

Plant materials. One hundred Dendrobium accessions were selected (Table 1). Thirtyseven of them originated from floricultural research institute, Guangdong Academy of Agricultural Sciences, China; the others came from Korea (three) and Japan (60). According to horticultural characters, they included 63 hybrid cultivars ( $\mathrm{c} 1$ to $\mathrm{c} 63$ ), six species of section Callista (c67, c71, c81, c82, c89, c94), six species of section Formosae (c72, c74, c76, c79, c88, c95), and 25 species of section Dendrobium (c64 to c66, c68 to c70, c73, c75, c77, c78, c80, c83 to c87, c90 to c93, c96 to $\mathrm{c} 100)$. Fresh leaf samples were collected from three individuals of each cultivar or species for genomic DNA extraction.

Genomic DNA extraction and amplified fragment length polymorphism reaction. Genomic DNA was extracted from leaf samples using a modified CTAB protocol as previously described (Li et al., 2007). AFLP assays and resolution of amplification products were performed according to the method of Zhu et al. (2007). Briefly, the restriction reaction was carried out with $200 \mathrm{ng}$ genomic DNA digested with EcoR I and Mse I. EcoR I and MseI adapters were ligated to the fragments. The adapter sequences, preselective amplification primers, and selective primers are listed in Table 2. Of these, eight primer pairs that generated well-defined patterns were chosen for use in this study (Table 3 ).

Data analysis. Generated AFLP bands were individually scored and statistically analyzed by following the assumption that fragment size as a locus was considered as either present (1) or absent (0) and finally 
constructed a binary matrix. Only fragments that had a molecular weight greater than 50 bps and high intensity were considered for data analysis. The Dice's similarity coefficient matrix was calculated using NTSYSpc package (Version 2.11) (Rohlf, 2004), and the dendrogram was produced using the UPGMA. The percent polymorphism was calculated as the ratio of total number of polymorphic bands compared with total number of bands.

\section{Results}

Amplified fragment length polymorphism profile and analysis. Eight selected primer combinations used in this study generated 1658 recordable bands with an average of 207 bands per primer pair, of which 1655 bands were polymorphic. The number of polymorphic bands varied from $185\left(\mathrm{E}_{\mathrm{ACA}}\right)$ $\left.\mathrm{M}_{\mathrm{CAG}}\right)$ to $215\left(\mathrm{E}_{\mathrm{ACT}} / \mathrm{M}_{\mathrm{CAA}}\right.$ or $\left.\mathrm{E}_{\mathrm{ACG}} / \mathrm{M}_{\mathrm{CTG}}\right)$. The number of polymorphic markers and percent polymorphism for each pair of primers are shown in Table 3. These data suggest that polymorphism of genomic DNA exists among native species and hybrid cultivars of Asian Dendrobium.

Amplified fragment length polymorphism markers for native species and hybrid cultivar diagnosis. Each of the tested Dendrobium accessions showed a unique AFLP genotyping profile based on measurement of 1655 AFLP polymorphic bands. Among eight AFLP primer pair combinations, they revealed amplified DNA fragments that were unique to 29 Dendrobium accessions (Table 1). These AFLP primers amplifying specific DNA fragments provide molecular tools for identification of tested native species and hybrids of Asian Dendrobium. For example, 5, 4, 3, 3, 3 and 3 AFLP markers were specific for D. sulcatum (c94), D. utopia 'Messenger' (c9), D. jenkinsii (c82), D. brymerianum (c85), D. aphyllum (c87), and D. densiflorum (c89), respectively. These data indicated that the AFLP technique was a useful molecular method for the identification of native species and hybrid cultivars of Asian Dendrobium at their genomic DNA levels.

Genetic relationships among native species and hybrids of Asian Dendrobium. The AFLP-generated data in this study were analyzed using Dice's similarity coefficient. According to the similarity matrix and UPGMA clustering, these native species and hybrid cultivars were grouped into seven clusters (I to VII) with a similarity level of 0.49 (Fig. 1). Cluster I consisted of 82 accessions and was further divided into five subclusters with a similarity level of 0.53 (Fig. 1): Subcluster Ia with 63 hybrid cultivars (c1 to c63) originated from Japan and Korea and three native species (c65, c66, and c98) belonging to section Dendrobium; Subcluster Ib with 12 native species (c68, c70, c74, c80, c85, c90, c91, c94, c96, c97, c99, and c100) belonging to section Dendrobium except two native species (c74 and c94); c74 and c94 belonging to sections Formosae and Callista, respectively; and Subclusters Ic, Id,
Table 1. List of plant materials used in the phylogenetic analysis of Dendrobium by amplified fragment length polymorphism (AFLP) markers.

\begin{tabular}{|c|c|c|c|c|c|c|}
\hline $\begin{array}{l}\text { Sample } \\
\text { no. }\end{array}$ & Species or cultivar & Section & Flower color & Origin & $\begin{array}{c}\text { Specific } \\
\text { (delete) } \\
\text { bands }\end{array}$ & $\begin{array}{l}\text { AFLP } \\
\text { cluster }\end{array}$ \\
\hline$\overline{\mathrm{c} 1}$ & D. Fairyflake 'Carmen' & Hybrid & Modena & Japan & 0 & Ia \\
\hline $\mathrm{c} 2$ & D. Spot Right & Hybrid & Modena & Japan & 0 & Ia \\
\hline $\mathrm{c} 3$ & D. Kazuki & Hybrid & Modena & Japan & 0 & Ia \\
\hline $\mathrm{c} 4$ & D. Yohkou 'Peach Boy' & Hybrid & Modena & Japan & 1 & Ia \\
\hline $\mathrm{c} 5$ & D. Yohkou 'Cat's Eyes' & Hybrid & Modena & Japan & 0 & Ia \\
\hline c6 & D. Full Bloom 'Hanahime' & Hybrid & Lilac & Japan & 0 & Ia \\
\hline c7 & D. Comet King 'Akatsuki' & Hybrid & Modena & Japan & 0 & Ia \\
\hline $\mathrm{c} 8$ & D. Pink Beauty 'Queen' & Hybrid & Modena & Japan & 0 & Ia \\
\hline c9 & D. Utopia 'Messenger' & Hybrid & Modena & Japan & 4 & Ia \\
\hline $\mathrm{c} 10$ & D. Tomofleak 'Napori' & Hybrid & Modena & Japan & 0 & Ia \\
\hline $\mathrm{c} 11$ & D. Maloflake 'Yumedono' & Hybrid & Modena & Japan & 0 & Ia \\
\hline $\mathrm{c} 12$ & D. Shinonome 'Karen' & Hybrid & Lilac & Japan & 0 & Ia \\
\hline $\mathrm{c} 13$ & D. 'Bizen Akebano' & Hybrid & Modena & Japan & 1 & Ia \\
\hline $\mathrm{c} 14$ & D. Casiflake & Hybrid & Modena & Japan & 0 & Ia \\
\hline $\mathrm{c} 15$ & D. Emur & Hybrid & Modena & Japan & 0 & Ia \\
\hline $\mathrm{c} 16$ & D. Tubuyaki & Hybrid & White & Japan & 0 & Ia \\
\hline $\mathrm{c} 17$ & D. Moon Harmaid & Hybrid & White & Japan & 0 & Ia \\
\hline $\mathrm{c} 18$ & D. Spring Color 'Hohoemi' & Hybrid & White & Japan & 0 & Ia \\
\hline $\mathrm{c} 19$ & D. Yaotome & Hybrid & Modena & Japan & 0 & Ia \\
\hline $\mathrm{c} 20$ & D. Red Arce & Hybrid & Modena & Japan & 1 & Ia \\
\hline $\mathrm{c} 21$ & D. Yukidaruma 'Queen' & Hybrid & White & Japan & 0 & Ia \\
\hline $\mathrm{c} 22$ & D. Love Story & Hybrid & White & Japan & 0 & Ia \\
\hline $\mathrm{c} 23$ & D. Payruroyzu & Hybrid & Lilac & Japan & 0 & Ia \\
\hline $\mathrm{c} 24$ & D. Sailor Boy 'Hatsuyuki' & Hybrid & White & Japan & 0 & Ia \\
\hline $\mathrm{c} 25$ & D. Second Love 'Tokimeki' & Hybrid & White & Japan & 0 & Ia \\
\hline $\mathrm{c} 26$ & D. 'Benikujaku' & Hybrid & Amaranth & Japan & 1 & Ia \\
\hline $\mathrm{c} 27$ & D. Oriental Sprit 'Rudoruph' & Hybrid & Garnet & Japan & 0 & Ia \\
\hline $\mathrm{c} 28$ & D. Sekand Rave 'Yakigi Pon' & Hybrid & White & Japan & 0 & Ia \\
\hline $\mathrm{c} 29$ & D. Shirasagi & Hybrid & White & Japan & 0 & Ia \\
\hline $\mathrm{c} 30$ & D. Marchen Color & Hybrid & White & Japan & 0 & Ia \\
\hline $\mathrm{c} 31$ & D. Rainbow 'Saira' & Hybrid & White & Japan & 0 & Ia \\
\hline $\mathrm{c} 32$ & D. Snowflake 'Otome' & Hybrid & White & Japan & 0 & Ia \\
\hline $\mathrm{c} 33$ & D. Himezakura 'Fujikka' & Hybrid & White & Japan & 0 & Ia \\
\hline $\mathrm{c} 34$ & D. Sping Color 'Green Sleep' & Hybrid & White & Japan & 0 & Ia \\
\hline $\mathrm{c} 35$ & D. Harugasumi & Hybrid & White & Japan & 0 & Ia \\
\hline $\mathrm{c} 36$ & D. Sailor Boy 'Elite' & Hybrid & White & Japan & 0 & Ia \\
\hline $\mathrm{c} 37$ & D. Pink Doll 'Elegance' & Hybrid & Pink & Japan & 0 & Ia \\
\hline $\mathrm{c} 38$ & D. Pittero Gold 'Princess' & Hybrid & Yellow-green & Japan & 1 & Ia \\
\hline c39 & D. Nice Dream 'Lady' & Hybrid & Unknown & Japan & 0 & Ia \\
\hline $\mathrm{c} 40$ & D. Yellow Magic 'Carnival' & Hybrid & Orange & Japan & 0 & Ia \\
\hline $\mathrm{c} 41$ & D. White Rabbit 'Sakurahime' & Hybrid & White & Japan & 0 & Ia \\
\hline $\mathrm{c} 42$ & D. Sunny Gold 'Harp' & Hybrid & Unknown & Japan & 0 & Ia \\
\hline $\mathrm{c} 43$ & D. Lucky Gal 'Emito' & Hybrid & Reseda & Japan & 0 & Ia \\
\hline $\mathrm{c} 44$ & D. Spring Zewel 'Miui' & Hybrid & Reseda & Japan & 0 & Ia \\
\hline $\mathrm{c} 45$ & D. Yourpin Red & Hybrid & Purple & Japan & 0 & Ia \\
\hline $\mathrm{c} 46$ & D. Okayama Gold 'Harmony' & Hybrid & Yellow & Japan & 0 & Ia \\
\hline $\mathrm{c} 47$ & D. Spring Dream 'Kumiko' & Hybrid & White & Japan & 0 & Ia \\
\hline $\mathrm{c} 48$ & D. Sea Mary 'Snow King' & Hybrid & White & Japan & 0 & Ia \\
\hline $\mathrm{c} 51$ & D. Yellow Song 'Rhythm' & Hybrid & Yellow & Japan & 0 & Ia \\
\hline $\mathrm{c} 52$ & D. Marohuiku & Hybrid & Modena & Japan & 0 & Ia \\
\hline $\mathrm{c} 53$ & D. Yohkou 'Cat's Eyes' & Hybrid & Garnet & Japan & 0 & Ia \\
\hline $\mathrm{c} 54$ & D. Lady Smile & Hybrid & Modena & Japan & 0 & Ia \\
\hline $\mathrm{c} 55$ & D. Stardust 'Fire Bird' & Hybrid & Orange & Japan & 0 & Ia \\
\hline $\mathrm{c} 56$ & D. Red Star & Hybrid & Purple & Japan & 0 & Ia \\
\hline $\mathrm{c} 57$ & D. Hamanal Lake 'Dream' & Hybrid & Purple & Japan & 0 & Ia \\
\hline $\mathrm{c} 58$ & D. Spring Jewel 'Miki' & Hybrid & White & Japan & 0 & Ia \\
\hline c59 & D. Yume Ji 'No.2' & Hybrid & Yellow & Japan & 0 & Ia \\
\hline $\mathrm{c} 60$ & D. 'Jin Shan' & Hybrid & White & Korea & 0 & Ia \\
\hline c61 & D. 'Yun Xue' & Hybrid & White & Korea & 0 & Ia \\
\hline $\mathrm{c} 62$ & D. 'Xu Yun' & Hybrid & White & Korea & 0 & Ia \\
\hline $\mathrm{c} 63$ & $D$. hybrid & Hybrid & White & Japan & 0 & Ia \\
\hline $\mathrm{c} 64$ & D. parishi & Dendrobium & Purple & China & 1 & II \\
\hline $\mathrm{c} 65$ & D. nobile & Dendrobium & White & China & 0 & Ia \\
\hline c66 & D. moniliforme variation & Dendrobium & Kelly & China & 0 & Ia \\
\hline $\mathrm{c} 67$ & D. thyrsiflorum & Callista & White & China & 1 & VI \\
\hline $\mathrm{c} 68$ & D. chrysanthum & Dendrobium & Yellow & China & 1 & $\mathrm{Ib}$ \\
\hline c69 & Unknown & Dendrobium & Unknown & China & 2 & VII \\
\hline $\mathrm{c} 70$ & D. fimbriatum & Dendrobium & Orange & China & 0 & $\mathrm{Ib}$ \\
\hline c71 & D. chrysotoxum & Callista & Golden & China & 2 & V \\
\hline c72 & D. williamsonii & Formosae & Primrose & China & 0 & III \\
\hline c73 & D. hancockii & Dendrobium & Yellow & China & 0 & II \\
\hline
\end{tabular}


Table 1. (Continued) List of plant materials used in the phylogenetic analysis of Dendrobium by amplified fragment length polymorphism (AFLP) markers.

\begin{tabular}{|c|c|c|c|c|c|c|}
\hline $\begin{array}{l}\text { Sample } \\
\text { no. }\end{array}$ & Species or cultivar & Section & Flower color & Origin & $\begin{array}{c}\text { Specific } \\
\text { (delete) } \\
\text { bands }\end{array}$ & $\begin{array}{l}\text { AFLP } \\
\text { cluster }\end{array}$ \\
\hline$\overline{\mathrm{c} 74}$ & D. trigonopus & Formosae & Golden & China & 0 & $\mathrm{Ib}$ \\
\hline c75 & D. guangxiense & Dendrobium & Primrose & China & 0 & VII \\
\hline c76 & D. cariniferum & Formosae & White & China & 0 & III \\
\hline c77 & D. pendulum & Dendrobium & White & China & 1 & IV \\
\hline c78 & D. capillipes & Dendrobium & Yellow & China & 1 & Ie \\
\hline c79 & D. christyanum & Formosae & White & China & 0 & III \\
\hline c80 & D. falconeri & Dendrobium & White & China & 0 & $\mathrm{Ib}$ \\
\hline c81 & D. lindleyi & Callista & Primrose & China & 1 & V \\
\hline c82 & D. jenkinsii & Callista & Yellow & China & 3 & V \\
\hline c83 & D. chryseum & Dendrobium & Yellow & China & 1 & Ic \\
\hline c84 & D. loddigesii & Dendrobium & Rosy & China & $1(1)$ & II \\
\hline $\mathrm{c} 85$ & D. brymerianum & Dendrobium & Yellow & China & 3 & $\mathrm{Ib}$ \\
\hline c86 & D. wardianum & Dendrobium & White & China & 2 & Id \\
\hline c87 & D. aphyllum & Dendrobium & Lilac & China & 3 & II \\
\hline c88 & D. bellatulum & Formosae & White & China & 0 & III \\
\hline c89 & D. densiflorum & Callista & Golden & China & 2 & VI \\
\hline c90 & D. devonianum & Dendrobium & White & China & 0 & $\mathrm{Ib}$ \\
\hline c91 & D. gibsonii & Dendrobium & Orange & China & 1 & $\mathrm{Ib}$ \\
\hline c92 & D. gratiosisimum & Dendrobium & Pink & China & 1 & Id \\
\hline c93 & D. primulinum & Dendrobium & Pink & China & 0 & II \\
\hline c94 & D. sulcatum & Callista & Yellow & China & 5 & $\mathrm{Ib}$ \\
\hline c95 & D. infundibulum & Formosae & White & China & 1 & III \\
\hline c96 & D. heterocarpum & Dendrobium & Yellow & China & 1 & $\mathrm{Ib}$ \\
\hline c97 & D. harveyanum & Dendrobium & Golden & China & 2 & $\mathrm{Ib}$ \\
\hline c98 & D. henryi & Dendrobium & Golden & China & 2 & Ia \\
\hline c99 & D. crystallinum & Dendrobium & White & China & 3 & $\mathrm{Ib}$ \\
\hline $\mathrm{c} 100$ & D. moniliforme & Dendrobium & Primrose & China & 0 & $\mathrm{Ib}$ \\
\hline
\end{tabular}

Table 2. Sequences of oligonucleotide adaptor and primers used in phylogenetic analysis of 100 Asian Dendrobium accessions by amplified fragment length polymorphism markers.

\begin{tabular}{|c|c|c|}
\hline Adaptor/primer & Code & Sequence $\left(5^{\prime} \rightarrow 3^{\prime}\right)$ \\
\hline \multicolumn{3}{|l|}{ Adaptors } \\
\hline \multicolumn{2}{|l|}{ EcoR I adaptor } & $\begin{array}{l}\text { CTC GTA GAC TGC GTA CC } \\
\text { AAT TGG TAC GCA GTC TAC }\end{array}$ \\
\hline Mse I adaptor & & $\begin{array}{l}\text { GAC GAT GAG TCC TGA G } \\
\text { TAC TCA GGA CTC AT }\end{array}$ \\
\hline \multicolumn{3}{|c|}{ Preselective amplification primers } \\
\hline EcoR I primer $+\mathrm{A}$ & & GAC TGC GTA CCA ATT CA \\
\hline Mse I primer $+\mathrm{C}$ & & GAT GAG TCC TGA GTA AC \\
\hline \multicolumn{3}{|c|}{ Selective amplification primers } \\
\hline$E c o R \mathrm{I}+\mathrm{AAC}$ & $\mathrm{E}_{\mathrm{AAC}}$ & GAC TGC GTA CCA ATT C AAC \\
\hline$E c o R \mathrm{I}+\mathrm{ACA}$ & $\mathrm{E}_{\mathrm{ACA}}$ & GAC TGC GTA CCA ATT C ACA \\
\hline$E c o R \mathrm{I}+\mathrm{ACT}$ & $\mathrm{E}_{\mathrm{ACT}}$ & GAC TGC GTA CCA ATT C ACT \\
\hline$E c o R \mathrm{I}+\mathrm{ACC}$ & $\mathrm{E}_{\mathrm{ACC}}$ & GAC TGC GTA CCA ATT C ACC \\
\hline$E c o R \mathrm{I}+\mathrm{ACG}$ & $\mathrm{E}_{\mathrm{ACG}}$ & GAC TGC GTA CCA ATT C ACG \\
\hline Mse I + CTG & $\mathrm{M}_{\mathrm{CTG}}$ & GAT GAG TCC TGA GTA A CTG \\
\hline Mse I + CAG & $\mathrm{M}_{\mathrm{CAG}}$ & GAT GAG TCC TGA GTA A CAG \\
\hline Mse I + CAA & $\mathrm{M}_{\mathrm{CAA}}$ & GAT GAG TCC TGA GTA A CAA \\
\hline Mse I + CTT & $\mathrm{M}_{\mathrm{CTT}}$ & GAT GAG TCC TGA GTA A CTT \\
\hline Mse I + CTC & $\mathrm{M}_{\mathrm{CTC}}$ & GAT GAG TCC TGA GTA A CTC \\
\hline
\end{tabular}

Table 3. Banding pattern information in DNA polymorphism of 100 Dendrobium accessions with amplified fragment length polymorphism markers.

\begin{tabular}{lccc}
\hline $\begin{array}{l}\text { Primer } \\
\text { combination }\end{array}$ & $\begin{array}{c}\text { No. of total } \\
\text { bands }\end{array}$ & $\begin{array}{c}\text { No. of } \\
\text { polymorphic bands }\end{array}$ & $\begin{array}{c}\text { Percent of } \\
\text { polymorphism }\end{array}$ \\
\hline $\mathrm{E}_{\mathrm{AAC}} / \mathrm{M}_{\mathrm{CTG}}$ & 215 & 213 & 99.1 \\
$\mathrm{E}_{\mathrm{ACA}} / \mathrm{M}_{\mathrm{CAG}}$ & 185 & 185 & 100 \\
$\mathrm{E}_{\mathrm{ACT}} / \mathrm{M}_{\mathrm{CAA}}$ & 215 & 215 & 100 \\
$\mathrm{E}_{\mathrm{ACT}} / \mathrm{M}_{\mathrm{CTT}}$ & 211 & 211 & 100 \\
$\mathrm{E}_{\mathrm{ACC}} / \mathrm{M}_{\mathrm{CTG}}$ & 197 & 197 & 100 \\
$\mathrm{E}_{\mathrm{ACC}} / \mathrm{M}_{\mathrm{CTT}}$ & 208 & 208 & 100 \\
$\mathrm{E}_{\mathrm{ACG}} / \mathrm{M}_{\mathrm{CTC}}$ & 211 & 211 & 100 \\
$\mathrm{E}_{\mathrm{ACG}} / \mathrm{M}_{\mathrm{CTG}}$ & 216 & 215 & 99.5 \\
Total & 1658 & 1655 & - \\
Mean & 207 & 206 & - \\
\hline
\end{tabular}

and Ie with one (c83), two (c86 and c92), and one (c78) native species, respectively, all of them belonging to section Dendrobium. Cluster II contained five native species (c72, c76, c79, c88, and c95), all of which belonged to section Dendrobium. Cluster III also included five native species (c72, c76, c79, c88, and c95) belonging to section Formosae. Clusters IV and VII contained one (c77) and two (c69 and c75) native species, respectively, all belonging to section Dendrobium. Clusters V and VI included three (c71, c81, and c82) and two (c67 and c89) native species, respectively, all belonging to section Callista.

\section{Discussion}

For breeding, it is important to evaluate the phylogenetic relationships among native species and hybrid cultivars of Asian Dendrobium. However, despite different molecular tools being used, the available information of genetic relationships among native species and hybrid cultivars of Asian Dendrobium is still very limited (Burke et al., 2008; Gu et al., 2007; Lau et al., 2001; Tsai et al., 2004; Wang et al., 2009; Wongsawad et al., 2001; Yuan et al., 2009; Yue et al., 2006; Yukawa, 2001; Yukawa et al., 1993, 1996). In the current study, AFLP markers were used to analyze genetic relationships and molecular phylogeny among 63 hybrid cultivars and 37 native species of Asian Dendrobium. Eight primer combinations generated a total of 1658 bands with $99.8 \%$ polymorphism. In genus Pistacia, AFLP markers were used for the determination of phylogenetic relationships among 50 genotypes, including 44 accessions belonging to subspecies of $P$. atlantica (atlantica, mutica, kurdica, and cabulica), three unknown genotypes, and three accessions proposed to be hybrid from $P$. eurycarpa $\times P$. atlantica (Karimi et al., 2009). Moreover, AFLP markers were successfully used to genetically classify cultivars or varieties of a bamboo species (Lin et al., 2009). Our results revealed that AFLP technique was a powerful tool for analyses of genetic relationships among native species and hybrid cultivars of Asian Dendrobium.

According to traditional morphological classification, 74 Dendrobium species and two varieties were classified into 12 sections (Tsi et al., 1999). In our AFLP clusters, Cluster II contained five species, all belonging to section Dendrobium, and Cluster III also included five species, all belonging to section Formosae (Fig. 1). These AFLP clusters were consistent with traditional classifications as described by Tsi et al. (1999). These results further indicated that morphological classifications are credible, to some extent. Meanwhile, our AFLP data were sometimes inconsistent with the morphological classifications. For example, all native species in Subcluster Ib belonged to section Dendrobium except c74 and c94. These results revealed that the two native species (c74 and c94) had a close relationship with section Dendrobium in Subcluster Ib. These discrepancies were useful supplements for the morphological classifications.

HortScience Vol. 46(2) February 2011 


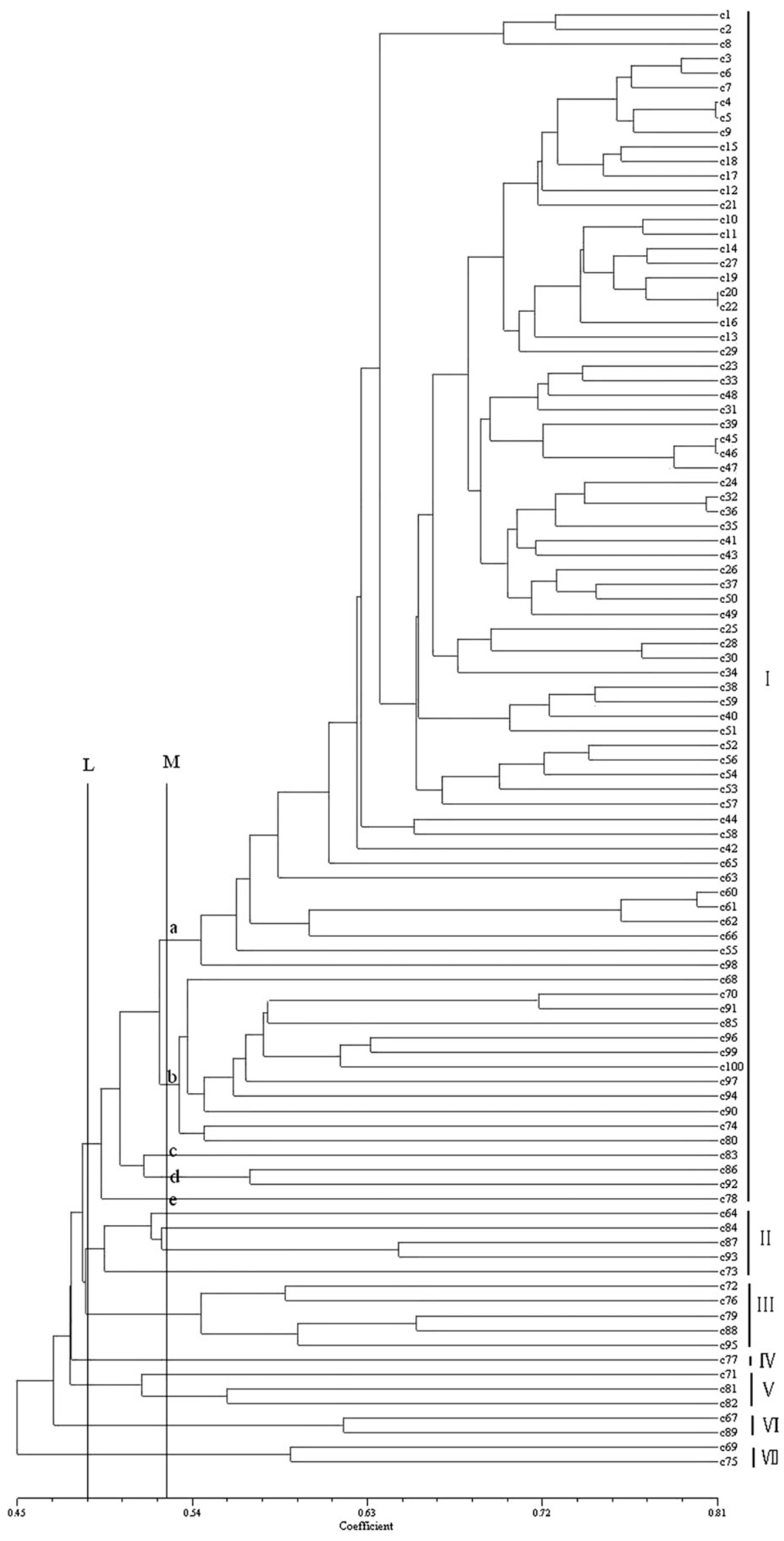

Fig. 1. Dendrogram constructed for 100 accessions based on unweighted pair group method based on unweighted pair group arithmetic average method (the same code numbers as listed in Table 1).
The AFLP classifications were to some extent related to flower colors of tested Asian Dendrobium accessions. For example, Cluster Ib contained 12 native species of Asian Dendrobium, all of which were yellow flowers or white flowers with yellow lips. These results were consistent with our analyses on genetic relationships among Cymbidium hybrids using random amplified polymorphic DNA markers (Li et al., 2007).

In this study, AFLP results indicated that the genetic relationships among tested Asian Dendrobium accessions were related to their origins and pedigree. On one hand, most hybrid cultivars of Dendrobium coming from Japan displayed close relationships, which were attributed to AFLP Cluster Ia (Fig. 1). On the other hand, three native species (c65, c66, and c98) were also classified into the same Cluster Ia (Fig. 1). These results revealed that these tested hybrid cultivars may be derived from the three native species (c65, c66, and c98). Among the hybrid cultivars, c10, c11, c14, c19, and c27 possessed the kin of D. snowflake 'Otome' (c32) (see Supplemental Fig. 1). However, these five hybrid cultivars and D. snowflake 'Otome' were classified into different subclusters in Ia (Fig. 1). The reason may be that these five tested hybrids not only gained the genetic background from $D$. snowflake 'Otome', but also acquired different genetic background from their the other parents. D. moniliforme variation (c66) and three hybrids (c60, c61, and c62) originating from Korea were classified into the same subcluster in Ia (Fig. 1). This result indicated that these three tested hybrids may be derived from a $D$. moniliforme variation.

We further found that 25 native species of section Dendrobium comprised at least four clusters (Fig. 1). Fifteen of these 25 Dendrobium species were the same species as used in ITS and matK classifications (Wongsawad et al., 2001). Our AFLP analysis was mostly consistent with molecular classifications by ITS and matK sequences (Wongsawad et al., 2001). For example, D. parishii, D. primulinum, D. aphyllum, and D. lodigesii were classified in the same cluster by both ITS and matK sequences and AFLP markers. However, considerable discrepancies were observed between these molecular results and ISSR classifications among 31 Dendrobium species (Wang et al., 2009). Therefore, multiple DNA molecular markers will show more accurate phylogenetic relationships among native species of Dendrobium.

In the present study, specific DNA markers were found in 29 of 100 tested Dendrobium accessions (Table 1). These were comparable with the species-specific ISSR markers discovered in nine of 31 investigated Dendrobium species (Wang et al., 2009). Transferring of these specific AFLP markers into sequencecharacterized amplified region markers would be very useful for molecular diagnosis of Dendrobium species and hybrid cultivars.

In conclusion, our study indicated that the AFLP tool was very effective in evaluating genetic relationships among native species and hybrid cultivars of Asian Dendrobium. 
Our AFLP cluster analysis clearly identified native species and hybrid cultivars of Asian Dendrobium and provided a molecular diagnosis tool for them. The AFLP markers reported in this study will accelerate understanding genetic structure, genetic diversity, and phylogenetic relationships of native species and hybrid cultivars of Asian Dendrobium.

\section{Literature Cited}

Bulpitt, C.J., Y. Li, P.F. Bulpitt, and J. Wang. 2007. The use of orchids in Chinese medicine. J. R. Soc. Med. 100:558-563.

Burke, J.M., M.J. Bayly, B. Peter, P.B. Adams, and P.Y. Ladiges. 2008. Molecular phylogenetic analysis of Dendrobium (Orchidaceae), with emphasis on the Australian section Dendrocor$y n e$, and implications for genetic classification. Aust. Syst. Bot. 21:1-14.

Chen, S.C. and Z.H. Tsi. 2000. The orchids of China. 2nd Ed. The Chinese Forestry Press, Beijing, China.

Depypere, L., P. Chaerle, P. Breyne, K.V. Mijnsbrugge, and P. Goetghebeur. 2009. A combined morphometric and AFLP based diversity study challenges the taxonomy of the European members of the complex Prunus L. section Prunus. Plant Syst. Evol. 279:219-231.

Gu, S., X.Y. Ding, Y. Wang, Q. Zhou, G. Ding, X.X. $\mathrm{Li}$, and L. Qian. 2007. Isolation and characterization of microsatellite markers in Dendrobium officinale, an endangered herb endemic to China. Mol. Ecol. Notes 7:1166-1168.

Karimi, H.R., S. Kafkas, Z. Zamani, A. Ebadi, and M.R. Fatahi Moghadam. 2009. Genetic relationships among Pistacia species using AFLP markers. Plant Syst. Evol. 279:21-28.

Lau, D.T.W., P.C. Shaw, J. Wang, and P.P.H. But. 2001. Authentication of medicinal Dendrobium species by the internal transcribed spacer of ribosomal DNA. Planta Med. 67:456-460.

Li, D., Q. Ye, and G. Zhu. 2007. Analysis of the germplasm resources and genetic relationships among hybrid Cymbidium cultivars and native species with RAPD markers. Agric. Sci. China 6:922-929.

Li, X., X. Ding, B. Chu, Q. Zhou, G. Ding, and S. Gu. 2008. Genetic diversity analysis and conservation of the endangered Chinese endemic herb Dendrobium officinale Kimura et Migo (Orchidaceae) based on AFLP. Genetica 133: $159-166$.

Lin, X.C., X.S. Ruan, Y.F. Lou, X.Q. Guo, and W. Fang. 2009. Genetic similarity among cultivars of Phyllostachys pubescens. Plant Syst. Evol. 277:67-73.

Pamidiamarri, D.V.N.S., N. Pandya, M.P. Reddy, and T. Radhakrishnan. 2009. Comparative study of interspecific genetic divergence and phylogenic analysis of genus Jatropha by RAPD and AFLP. Mol. Biol. Rep. 36:901-907.

Rohlf, J.F. 2004. NTSYS-pc: 2.11 Numerical Taxonomy and Multivariate Analysis System. Version 2.11. Exeter Publishing Ltd, New York, NY.

Seehalak, W., N. Tomooka, A. Waranyuwat, P. Thipyapong, P. Laosuwan, A. Kaga, and D.A. Vaughan. 2006. Genetic diversity of the Vigna germplasm from Thailand and neighboring regions revealed by AFLP analysis. Genet. Resources Crop Evol. 53:1043-1059.

Tsai, C.C., C.I. Peng, S.C. Huang, P.L. Huang, and C.H. Chou. 2004. Determination of the genetic relationship of Dendrobium species (Orchidaceae) in Taiwan based on the sequence of the internal transcribed spacer of ribosomal DNA. Sci. Hort. 101:315-325.

Tsi, Z.H., S.C. Chen, Y.B. Luo, and G.H. Zhu. 1999. Orchidaceae (3). In: Tsi, Z.H. (ed.). Angiospermae, Monocotyledoneae, Flora reipublicae popularis sinicae. Vol. 19. Sci. Press, Beijing, China.
Vos, P., R. Hogers, M. Bleeker, M. Reijans, V. de Lee, T. Miranda, F.A. Hornes, J. Pot, J. Peleman, M. Kuiper, and M. Zabeau. 1995. AFLP: A new technique for DNA fingerprinting. Nucleic Acids Res. 23:4407-4414.

Wang, H.Z., S.G. Feng, J.J. Lu, N.N. Shi, and J.J. Liu. 2009. Phylogenetic study and molecular identification of 31 Dendrobium species using inter-simple sequence repeat (ISSR) markers. Sci. Hort. 122:440-447.

Wongsawad, P., T. Handa, and T. Yukawa. 2001. Molecular phylogeny of Dendrobium section Dendrobium. Proc. 7th Asia Pacific Orchid Conf. p. 209-210.

Yuan, Z.Q., J.Y. Zhang, and T. Liu. 2009. Phylogenetic relationship of China Dendrobium species based on the sequence of the internal transcribed spacer of ribosomal DNA. Biol. Plant. 53:155-158.

Yue, G.H., L.T. Lam-Chan, and Y. Hong. 2006. Development of simple sequence repeat (SSR) markers and their use in identification of Dendrobium varieties. Mol. Ecol. Notes 6:832834.

Yukawa, T. 2001. Molecular phylogeny of Dendrobium. Proc. 7th Asia Pacific Orchid Conf. p. 69-71.

Yukawa, T., S. Kurita, M. Nishida, and M. Hasebe. 1993. Phylogenetic implications of chloroplast DNA restriction site variation in subtribe Dendrobiinae (Orchidaceae). Lindlayana 8:211221.

Yukawa, T., H. Ohba, K.M. Cameron, and M.W. Chase. 1996. Chloroplast DNA phylogeny of subtribe Dendrobiinae (Orchidaceae): Insights from a combined analysis base on rbcL sequences and restriction site variation. J. Plant Res. 109:169-176.

Zhu, G., D. Li, and Z. Guo. 2007. Genetic diversity and relationship of hybrid Cymbidium based on AFLP marker. Acta Hort. Sinica 34:417-424 [with English abstr.]. 